\title{
The Hybrid Micro-chaos Map: Digitally Controlled Inverted Pendulum with Dry Friction
}

\author{
Gergely Gyebrószki1*, Gábor Csernák² \\ 1 Department of Applied Mechanics, Faculty of Mechanical Engineering, Budapest University of Technology and Economics, \\ H-1111 Budapest, Múegyetem rkp. 3., Hungary \\ 2 MTA-BME Research Group on Dynamics of Machines and Vehicles, H-1111 Budapest, Müegyetem rkp. 3., Hungary \\ * Corresponding author, e-mail: gyebro@mm.bme.hu
}

Received: 13 October 2018, Accepted: 21 November 2018, Published online: 11 February 2019

\begin{abstract}
Digital effects (quantization, sampling and delay) can lead to small amplitude chaotic oscillations, called micro-chaos [1, 2]. Often, these vibrations are neglected due to their small amplitude or replaced by random noise, but doing so one might be unable to capture some important behavior of the digitally controlled system.

One notable example is the PD-controlled inverted pendulum with quantization at the calculated control effort. Taking digital effects into account leads to separated chaotic attractors in the state space. While the amplitude of the chaotic oscillations is indeed small, these attractors are situated rather far from the desired position, introducing considerable control error. Micro-chaos is undoubtedly present in ideal models of computer-controlled mechanical systems, however an important question is still open: does it persist if a more complex model of reality is used? For instance, does it survive in the presence of dry friction?

This paper answers the latter question analyzing the micro-chaos in a system with Coulomb friction. We introduce the so-called hybrid micro-chaos map that describes the behavior of a digitally controlled system with dry friction. Then, the theoretical analysis of this map is presented and numerical results are provided that were acquired using a new mathematical tool, the Clustered Simple Cell Mapping method.

Lastly, we conclude, that the phenomena of micro-chaos can withstand the presence of Coulomb friction and chaotic attractors can coexist with sticking zones in the state space.
\end{abstract}

Keywords

micro-chaos, Coulomb friction, quantization, rounding

\section{Introduction}

In our past works, we have analyzed various digitally controlled systems exhibiting micro-chaos: small amplitude chaotic oscillations. In case of quantization at the control effort (output of the controller), separated chaotic attractors are present in the state-space [3]. However, if the measured states (input of the controller) are quantized, usually a periodic orbit appears with superimposed chaotic oscillation [4].

Micro-chaos also appears in other research areas beyond digital control. One notable example is the field of human postural balance, where sensory dead zones, sampled and time-delayed feedback of the neural control results in micro-chaotic oscillations $[5,6]$. The model of inverted pendulum has a great significance in this area, as well, during the analysis of stick-balancing [6] which can provide important results to understand the control strategy implemented by the human brain when compared with measurements. As it was presented in [7], quantization can contribute to the stabilization of unstable dynamical systems in the presence of feedback delay in neural control.

The present paper is mainly based on the results of [8], where the dynamics of a digitally controlled inverted pendulum was analyzed without dry friction. As it was proved, sampling and quantization leads to chaotic behavior and the appearance of coexisting chaotic attractors and repellors in the phase-space of the considered system. The finer structure of these strange objects was discussed in [9], where an estimate was given for the maximal quantization-related control error.

While sampling and delay are commonly considered in control problems, rounding is usually neglected, or replaced 
by quantization noise [10]. Even though we have shown that properly taking quantization into account results in different behavior compared to the neglection or replacement by random-noise approach, we have not addressed an important question yet: Does micro-chaos persist if friction is present?

This paper introduces the hybrid micro-chaos map, where the term hybrid refers to the two types of switching in the system: the map-like switching of the control effort that happens only at sampling time instants and the flow-like, continuous switching of the friction force at the sign changes of the velocity. We present simple analytical formulas to determine if chaotic attractors are affected by the sticking introduced by the friction or not. Finally, we present numerical results obtained using Clustered Simple Cell Mapping [11] to support our theoretical results.

\section{PD-controlled inverted pendulum with dry friction}

Consider an inverted pendulum controlled according to a proportional-derivative (PD) scheme with sampling and zero- order hold (i.e., the control effort is kept constant between two successive sampling instants) as shown in Fig. 1. Dry friction, symbolized by $\mu$ in the figure, is temporarily omitted.

The equation of motion of the frictionless system is

$$
\begin{aligned}
J \ddot{\varphi}(t) & =m g h \sin (\varphi(t))-k \dot{\varphi}(t)-p \varphi_{i}-d \dot{\varphi}_{i} \\
t & \in[i \tau,(i+1) \tau),
\end{aligned}
$$

where $m$ and $J$ are the mass and mass moment of inertia of the pendulum, $h$ is the distance between the center of mass and the axis of rotation, $p$ and $d$ are control gains and $k$ is the linear damping coefficient. Gravitational acceleration is denoted by $g, \tau$ is the sampling time, while $\varphi_{i}=\varphi(i \tau)$ and $\dot{\varphi}_{i}=\dot{\varphi}(i \tau)$ are sampled values of the angular position and angular velocity, respectively (at the beginning of the $i^{\text {th }}$ time interval). Rearranging and linearizing Eq. (1) yields $\ddot{\varphi}(t)+2 \alpha \delta \dot{\varphi}(t)-\alpha^{2} \varphi(t)=-P \varphi_{i}-D \dot{\varphi}_{i}$,

where $\alpha^{2}=m g h / J, 2 \alpha \delta=k / J, P=p / J, D=d / J$.

As we have shown in [8], if the control torque can assume only quantized values, this system exhibits small amplitude chaotic vibrations. However, as it was pointed out in [12], Coulomb friction has a major role in the damping of such systems. Therefore, our present goal is to analyze the effect of dry friction on the previously found, so-called micro-chaotic solutions. Before formulating the equations for the quantized case, we add a new term to Eq. (2) that corresponds to Coulomb friction (see Fig. 2):

$\ddot{\varphi}(t)+2 \alpha \delta \dot{\varphi}(t)-\alpha^{2} \varphi(t)=-P \varphi_{i}-D \dot{\varphi}_{i}-\operatorname{sgn}(\dot{\varphi}(t)) \mu_{0}$.
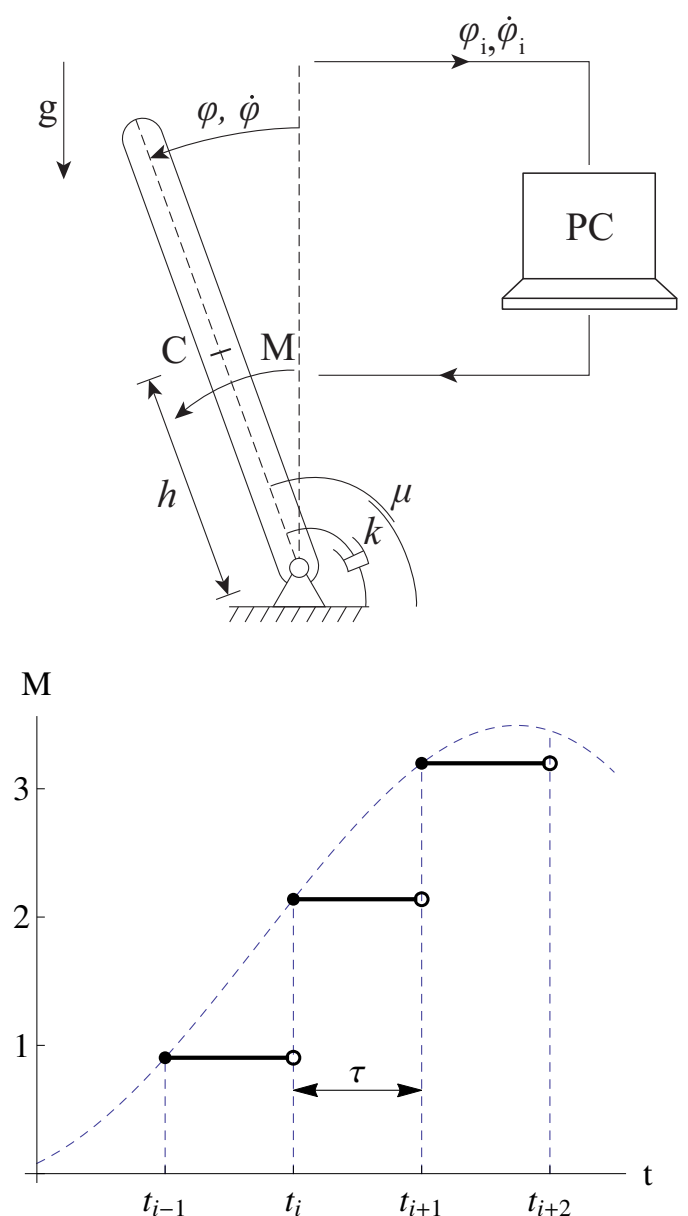

Fig. 1 Digitally controlled inverted pendulum realizing zero-order hold and the control torque $M$ with respect to time. Blue dashed envelope curve indicates a reference control torque corresponding to a continuous, non-sampled case.

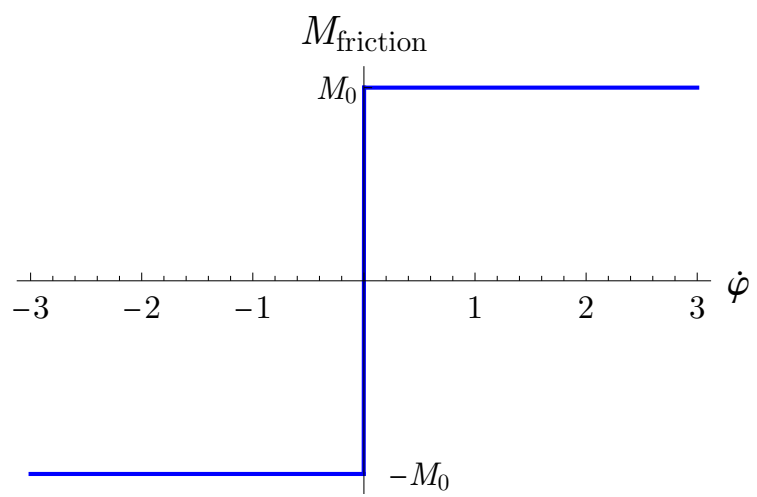

Fig. 2 Bearing friction with respect to angular velocity of the pendulum. The coefficients of kinetic and static friction are considered to be equal. 
Note, that this equation is non-linear due to the sgn function that may assume any value between -1 and 1 at zero angular velocity. The actual value of the friction force at $\dot{\varphi}(t)=0$ is determined by the other forces and torques acting on the pendulum. As a consequence, the upper equilibrium point turns to an interval of possible equilibrium positions. Thus, Eq. (3) cannot be linearized at the $\varphi=0, \dot{\varphi}=0$ state in the conventional manner. To linearize the equation in a mathematically correct way, one could apply the theory of differential inclusions [13] or introduce a regularized, smooth approximation to the sgn function. This latter approach was followed in [12].

However, as it will be shown in this section, quantization is another non-smooth effect that introduces non-linearity in the system. Thus, instead of focusing on the linearization process, we opted for using non-smooth models describing the effects of friction and quantization. It is usual in textbooks and papers dealing with dry friction (see e.g., [14] and [15]) that a friction force-related term $F_{\text {friction }}=-\operatorname{sgn}(v) F_{N}$ is added to otherwise linear(ized) equations, and the resulting piecewise linear system is analyzed. Even in case of time-delayed systems, piecewise smooth control input is considered similarly, [16]. Since this method led to qualitatively correct findings, it is often applied in the industry, too [17]. Following this approach, we continue the analysis of Eq. (3) without further linearization.

Equation (3) can be rewritten as a system of first order differential equations:

$$
\begin{aligned}
& \dot{\omega}(t)=\alpha^{2} \varphi(t)-2 \alpha \delta \omega(t)-P \varphi_{i}-D \omega_{i}-\operatorname{sgn}(\omega(t)) \mu_{0}, \\
& \dot{\varphi}(t)=\omega(t), \quad t \in[i \tau,(i+1) \tau),
\end{aligned}
$$

with initial conditions $\omega(i \tau)=\omega_{i}$ and $\varphi(i \tau)=\varphi_{i}$.

If the sign of the angular velocity does not change, Eq. (4) can be easily solved for a sampling interval. Introducing the notations $\hat{\alpha}=\alpha \tau, \Gamma=\sqrt{1+\delta^{2}}, \hat{P}=P \tau^{2}$, $\hat{D}=D \tau, x=\varphi /\left(r_{\text {out }} \tau^{2}\right), v=\omega /\left(r_{\text {out }} \tau\right), \hat{\mu}=\mu_{0} \tau^{2} /\left(r_{\text {out }} \tau^{2}\right)$, dimensionless time $T=t / \tau$ and $r_{\text {out }}$ as the resolution of the actuated control effort, the solution formulates a dimensionless 2D map:

$$
\begin{aligned}
& \mathbf{y}(T) \equiv[x(T) \quad v(T)]^{T}=\cdots \\
& =\mathbf{U}(T) \mathbf{y}(0)+\mathbf{b}(T) F(\mathbf{y}(0)), \quad T \in[0,1),
\end{aligned}
$$

where $F$ is composed of the control effort and friction force

$F(\mathbf{y}(T))=\hat{P} x(0)+\hat{D} v(0)+\operatorname{sgn}(v(T)) \hat{\mu}$

and the following are the solution operators of the ODE:

$$
\begin{aligned}
& \mathbf{U}(T)=\frac{e^{-\hat{\alpha} \cdot \delta \cdot T}}{\Gamma} \\
& .\left[\begin{array}{cc}
\Gamma \operatorname{ch}(\hat{\alpha} \Gamma T)+\delta \operatorname{sh}(\hat{\alpha} \Gamma T) & \operatorname{sh}(\hat{\alpha} \Gamma T) / \hat{\alpha} \\
\hat{\alpha} \operatorname{sh}(\hat{\alpha} \Gamma T) & \Gamma \operatorname{ch}(\hat{\alpha} \Gamma T)-\delta \operatorname{sh}(\hat{\alpha} \Gamma T)
\end{array}\right],
\end{aligned}
$$

and

$$
\mathbf{b}(T)=\frac{1}{\hat{\alpha}^{2} \Gamma}\left[\begin{array}{c}
\Gamma-e^{-\hat{\alpha} \delta T}(\Gamma \operatorname{ch}(\hat{\alpha} \Gamma T)+\delta \operatorname{sh}(\hat{\alpha} \Gamma T)) \\
-\hat{\alpha} e^{-\hat{\alpha} \delta T} \operatorname{sh}(\hat{\alpha} \Gamma T)
\end{array}\right] .
$$

The arguments of $\mathbf{U}$ and $\mathbf{b}$ refer to the length of the solution segment, while the argument of $\mathbf{y}$ refers to a specific dimensionless time instant.

\section{The hybrid micro-chaos map}

The calculated control force does not change within the sampling interval, due to the zero-order hold. Consequently, Eq. (5) can be used as a map between states at the $i^{\text {th }}$ and $(i+1)^{\mathrm{st}}$ sampling instants, if the sign of the velocity $v$ does not change either in the interval $T \in[i, i+1)$ :

$\mathbf{y}(i+T)=\mathbf{U}(T) \mathbf{y}(i)+\mathbf{b}(T) F(\mathbf{y}(i)), \quad T \in[0,1)$.

Introducing the notation $\mathbf{y}_{i}=\left[\begin{array}{ll}x(i) & v(i)\end{array}\right]^{T}$, the state vector at the next sampling instant can be expressed by substituting $T=1$ in Eq. (7). Thus, the following map is obtained:

$\mathbf{y}_{i+1}=\mathbf{U} \mathbf{y}_{i}+\mathbf{b}(\underbrace{\operatorname{Int}\left(\hat{P} x_{i}+\hat{D} v_{i}\right)}_{m_{i}}+\underbrace{\operatorname{sgn}\left(v_{i}\right) \hat{\mu}}_{\mu_{i}})$.

Here $m_{i}$ is the control effort, $\mu_{i}$ is the friction force and $\operatorname{Int}(\cdot)$ denotes the integer part function representing the quantization of the control effort. This quantization is a map-like switching in the system: the controller updates the control effort at sampling instants, only. At $\hat{\mu}=0$, there is no other discontinuity in the system, so Eq. (7) that can be referred to as micro-chaos map in this case [8] - fully describes the evolution of the solutions.

The signum function corresponding to the friction, however, is a flow-like switching, because the sign of the friction force changes at any time instant when the velocity changes sign - regardless of the sampling. To handle the case when the velocity changes sign within the $i^{\text {th }}$ sampling interval, we need to calculate the dimensionless time $T_{0, i}$ when the solution reaches the switching line of the friction force $\Sigma_{f}: \mathbf{y} \mid v=0$, i.e., when the velocity is zero. Expressing the velocity using Eq. (7), one arrives at

$\underbrace{\hat{\alpha} \Gamma v_{i}}_{:=C_{i}} \cosh \left(\hat{\alpha} \Gamma T_{0, i}\right)+$

$\underbrace{-\left(\hat{\alpha} \cdot \delta v_{i}-\hat{\alpha}^{2} x_{i}+m_{i}+\mu_{i}\right)}_{:=S_{i}} \sinh \left(\hat{\alpha} \Gamma T_{0, i}\right)=0$. 
From Eq. (9), the dimensionless time corresponding to zero velocity can be obtained:

$T_{0, i}=\frac{1}{\hat{\alpha} \Gamma} \log \left(\sqrt{\frac{S_{i}-C_{i}}{S_{i}+C_{i}}}\right)$,

where $S_{i}$ and $C_{i}$ are the coefficients of $\sinh \left(\hat{\alpha} \Gamma T_{0, i}\right)$ and $\cosh \left(\hat{\alpha} \Gamma T_{0, i}\right)$, respectively in Eq. (9). Both $S_{i}$ and $C_{i}$ depend on the state of the system (i.e., $x_{i}, v_{i}$ or $\mu_{i}$ ) at the beginning of the sampling interval. Thus, the state vector $\tilde{\mathbf{y}}_{i}$ must be supplemented with the friction state $\mu_{i}$ : $\tilde{\mathbf{y}}_{i}=\left[\begin{array}{lll}x_{i} & v_{i} & \mu_{i}\end{array}\right]^{T}$.

The condition of crossing the switching line of the friction force $\left(\Sigma_{f}\right)$ within the actual sampling interval is

$0 \leq T_{0, i}<1$.

If $T_{0, i}>1$, one can use Eq. (7). Otherwise, the mapping between successive sampling instants must be divided into three steps:

1. time evolution until the velocity becomes zero,

2. change of the sign of friction force (or sticking), and

3. time evolution until the next sampling interval.

Combining these steps, we can formulate the so-called hybrid micro-chaos map:

$$
\begin{aligned}
\tilde{\mathbf{y}}\left(T_{0, i}\right) & =\tilde{\mathbf{U}}\left(T_{0, i}\right) \tilde{\mathbf{y}}_{i}+\tilde{\mathbf{b}}\left(T_{0, i}\right) F\left(\tilde{\mathbf{y}}_{i}\right), \\
\tilde{\mathbf{y}}\left(T_{0, i}\right)^{+} & =\tilde{\mathbf{Q}} \tilde{\mathbf{y}}\left(T_{0, i}\right), \\
\tilde{\mathbf{y}}_{i+1} & =\tilde{\mathbf{U}}\left(1-T_{0, i}\right) \tilde{\mathbf{y}}\left(T_{0, i}\right)^{+}+\tilde{\mathbf{b}}\left(1-T_{0, i}\right) F\left(\tilde{\mathbf{y}}\left(T_{0, i}\right)^{+}\right),
\end{aligned}
$$

where

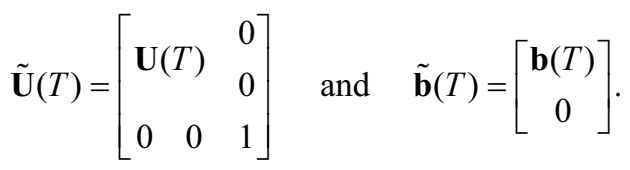

The discontinuity map related to the change of sign of the friction force is

$\tilde{\mathbf{Q}}= \begin{cases}{\left[\begin{array}{ccc}1 & 0 & 0 \\ 0 & 0 & 0 \\ 0 & 0 & 0\end{array}\right]} & \text { if sticking occurs, } \\ {\left[\begin{array}{ccc}1 & 0 & 0 \\ 0 & 1 & 0 \\ 0 & 0 & -1\end{array}\right] \quad \text { otherwise, }}\end{cases}$

where the condition of sticking is:

$\operatorname{abs}\left(\operatorname{Int}\left(P x_{i}+D v_{i}\right)-\alpha^{2} x\left(i+T_{0, i}\right)\right)<\mu$.

In the first case of Eq. (14), when Eq. (15) is satisfied, the solution sticks and will stay at $\Sigma_{f}$ Otherwise, the solution crosses the switching line and the sign of the friction force is changed. $\tilde{\mathbf{y}}\left(T_{0, i}\right)$ is the state when the solution reaches $\Sigma_{f}$ and $\tilde{\mathbf{y}}\left(T_{0, i}\right)^{+}$is the state immediately after crossing $\Sigma_{f}$.

It should be noted, that the initial state for map Eq. (12) should contain a friction force $\mu_{0}=\operatorname{sgn}\left(v_{0}\right) \hat{\mu}$, compatible with the initial velocity.

\section{Topological patterns, sticking zones}

The equation of control effort switching lines is $\operatorname{Int}(\hat{P} x+\hat{D} v)=m \Rightarrow v=\frac{m-\hat{P} x}{\hat{D}}, \quad m \in \mathbb{Z} \backslash\{0\}$.

The unstable fixed points $\mathbf{F}_{m}=\left[\begin{array}{cc}x_{u}^{m} & 0\end{array}\right]^{T}$ of the microchaos map lie on the $x$-axis, at zero velocity. If we omit the friction force,

$\operatorname{Int}\left(\hat{P} x_{u}^{m}\right)=\hat{\alpha}^{2} x_{u}^{m} \Rightarrow x_{u}^{m}=\frac{m}{\hat{\alpha}^{2}}, \quad m \in \mathbb{Z}$.

This formula is valid only if $x_{u}{ }^{m}$ resides between the $m^{\text {th }}$ and $(m+1)^{\text {st }}$ switching lines - since this is required to have $\operatorname{Int}\left(\hat{P} x_{u}^{m}\right)=m$. Based on Eqs. (16)-(17), unstable fixed points and switching lines occur alternately on the $x-v$ phase-space (see Fig. 3).

If unstable fixed points exist on both sides of a switching line, they push the trajectories towards each other. Therefore, chaotic attractors are expected to be at the intersections of the switching lines and the $x$ axis (see Fig. 3):

$x_{\text {attr }}=\frac{m}{\hat{P}}, \quad m \in \mathbb{Z} \backslash\{0\}$.

Restricting the control parameters to the stable domain $\left(\hat{P}>\hat{\alpha}^{2}\right)$, the index of the outermost attractor can be expressed as

$l_{\max }=\operatorname{Int}\left(\frac{\hat{P}}{\hat{P}-\hat{\alpha}^{2}}\right)$.

At $m>l_{\text {max }}$, the alternating pattern of attractors and fixed points breaks because the $m=l_{\text {max }}$ control effort band does not have a fixed point inside. This particular fixed point is virtual and is situated in the next control effort band, see Fig. 3. While there can exist an attractor between a real and a virtual fixed point [8], there cannot be attractors at switching lines if $m>l_{\max }$. Thus, one can expect to have several attractors when the difference between the control gain $\hat{P}$ and $\hat{\alpha}^{2}$ is relatively small. At certain parameter combinations (see [9]), the neighboring attractors merge and form a larger attractor.

An upper estimate for the control error - the distance of the outermost attractor from the origin - can be given for that frictionless case, when the eigenvalues of $\mathbf{U}$ are positive and real [9]: 


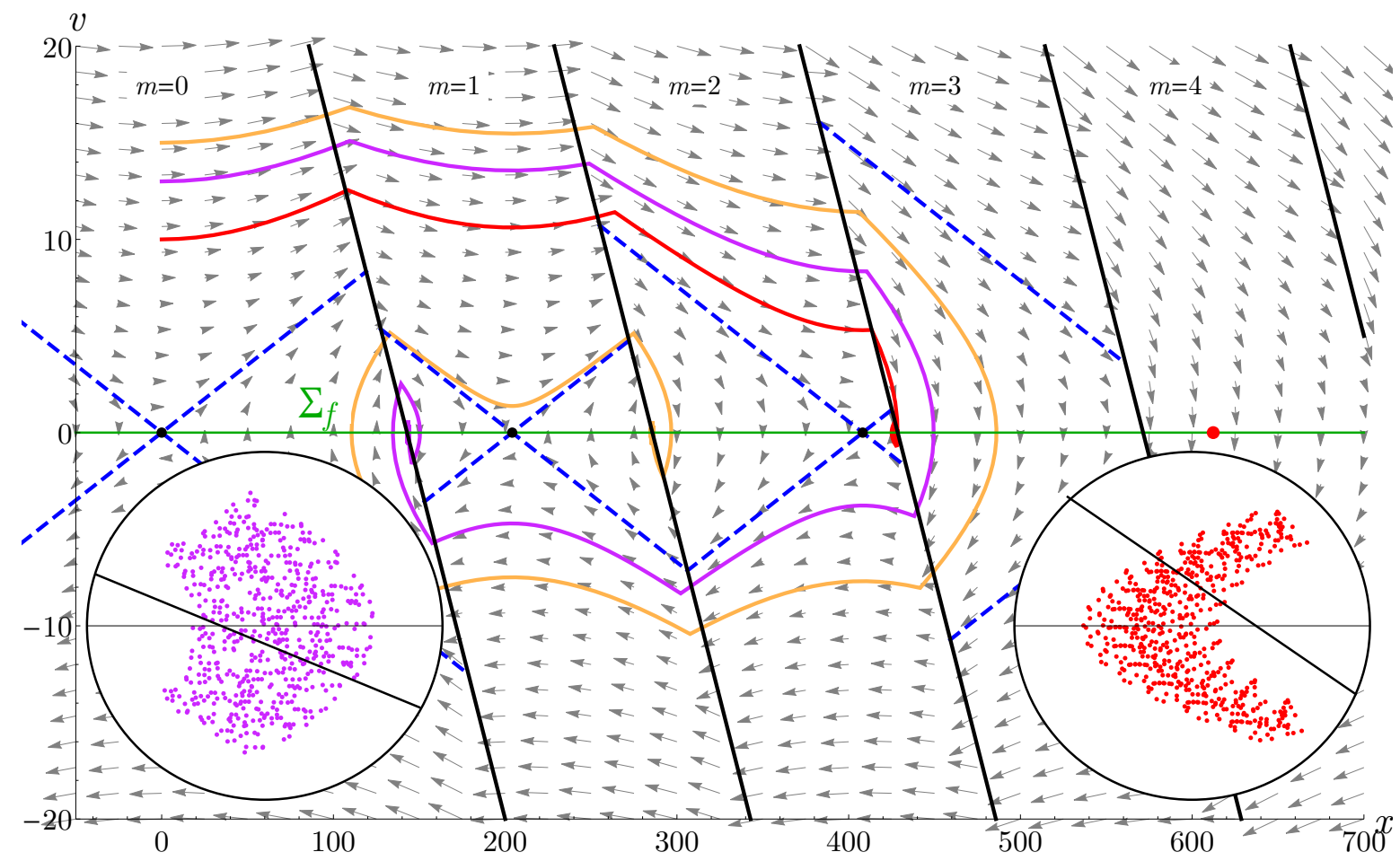

Fig. 3 The state space of the micro-chaos map, when $\hat{\mu}=0$. Black lines are control effort switching lines, $\Sigma_{f}$ is denoted with a green line, unstable fixed points are denoted with black dots and their manifolds are the blue dashed lines. Three example trajectories corresponding different initial velocities are shown, ending in different chaotic attractors. Here $l_{\max }=3$ and after the $3^{\text {rd }}$ attractor, the alternating pattern of attractors and fixed points breaks. The upcoming, virtual fixed point is shown with a red dot.

$x_{e r r, \max }=\frac{1}{\hat{P}-\hat{\alpha}^{2}}$.

As friction is introduced in the system, the fixed points extend to larger intervals along the $x$ axis, where the friction force can be in equilibrium with the other forces acting on the system. If such an equilibrium interval collides with an attractor, a crisis phenomenon occurs and the attractor turns to a repellor, exhibiting finite time transient chaotic behavior.

As it was shown in [9], there is only one fixed point in the phase space - at the origin - if large control gain $\hat{P}>2 \hat{\alpha}^{2}$ is chosen from the domain of stability. The corresponding large attractor immediately disappears if friction is present.

However, at relatively small values of $\hat{P}$ - when it is only slightly larger than $\hat{\alpha}^{2}-$, several small attractors coexist and some of them may be quite far from the neighboring fixed points. Thus, such attractors can persist even if friction is taken into account.

If we consider the condition of sticking Eq. (15) and substitute the control effort value $m$, we arrive at $\operatorname{abs}\left(m-\hat{\alpha}^{2} x\right)<\hat{\mu}$.
Resolving the absolute value yields

$\frac{m-\hat{\mu}}{\hat{\alpha}^{2}}<x_{\text {sticking }}<\frac{m+\hat{\mu}}{\hat{\alpha}^{2}}$.

One can recognize here the term corresponding to the unstable fixed points (Eq. (17)), that is, the $m^{\text {th }}$ sticking zone will appear around the $m^{\text {th }}$ unstable fixed point:

$x_{\text {sticking }} \in\left[x_{u, m}-\frac{\hat{\mu}}{\hat{\alpha}^{2}}, \quad x_{u, m}+\frac{\hat{\mu}}{\hat{\alpha}^{2}}\right]$.

Using Eq. (18) and Eq. (22), one can express the condition corresponding to the overlap of attractor $m$ and one of its neighboring sticking zones.

$\frac{m-1+\hat{\mu}}{\hat{\alpha}^{2}}=\frac{m}{\hat{P}} \quad$ or $\quad \frac{m}{\hat{P}}=\frac{m-\hat{\mu}}{\hat{\alpha}^{2}}$.

Lastly, one can also express the critical friction parameter when all sticking zones merge with their neighbors, that is the whole $x$-axis behaves as a sticking zone:

$\frac{\hat{\mu}_{c r i t}}{\hat{\alpha}^{2}}=\frac{1}{\hat{\alpha}^{2}} \Rightarrow \hat{\mu}_{\text {crit }}=1$.

Since the collision of chaotic attractors and sticking zones also depends on the size of the attractors, providing an exact formula for the absorption of a certain chaotic attractor can be challenging. Therefore, we have used cell 
mapping to examine the effect of increasing the friction parameter $\hat{\mu}$.

\section{Cell mapping results}

Cell mapping methods are suitable for the global investigation of the long term behavior of nonlinear dynamical systems [18]. Using cell mapping methods, fixed points, periodic orbits and their basin of attraction can be quickly found. Chaotic attractors are usually covered with one or more high-period orbits. We have applied Clustered Simple Cell Mapping (C-SCM) [11] to Eq. (12) to determine whether the chaotic attractors disappear due to the dry friction or not. Clustered Simple Cell Mapping is an extended variant of the Simple Cell Mapping (SCM) method. It works by solving separated SCM problems on adjacent state space regions and joining them to form a cluster of solutions. This way, adaptive expansion of the state space (by following trajectories leaving the state space domain covered by the cluster), and parallel execution are possible.

Consider Fig. 4, showing chaotic attractors (black dots), their basins of attraction (colored bands), switching lines (white), and manifolds of fixed points (dashed white lines). Results with $\hat{\mu}=0$ and $\hat{\mu}^{\prime}=0.095$ are shown, where the latter corresponds to the collision of the sticking zone and the $4^{\text {th }}$ attractor: $-1+\hat{\mu}^{\prime} / \alpha^{2}=m / P$, with $m=4$. Since domains of attraction are provided by cell mapping, one can clearly see which initial states lead to sticking zones (gray regions).

In order to explore the effect of varying $\hat{\mu}$, we have carried out a parameter scan on $\hat{\mu} \in[0,0.5]$. Attractor and fixed point positions (mean of $x$ coordinates) are shown in Fig. 5, with colored and gray dots, respectively. As the sticking zones around fixed points grow, they absorb chaotic attractors one after the other. In this particular case, all chaotic attractors are absorbed at $\hat{\mu} \approx 0.45$.

\section{Conclusion}

We have extended the notion of micro-chaos with the possibility to incorporate flow-like switching into a digitally controlled system. We introduced the hybrid microchaos map, and analyzed the behavior of a PD-controlled inverted pendulum with sampling and dry friction. Without friction, this system can have multiple separated chaotic attractors in its state space. Using the corresponding hybrid micro chaos map, the effect of friction was analyzed, and conditions for the collision of sticking zones and chaotic attractors could be given.

We have shown, that there is a wide range of parameters, where chaotic attractors coexist with sticking zones
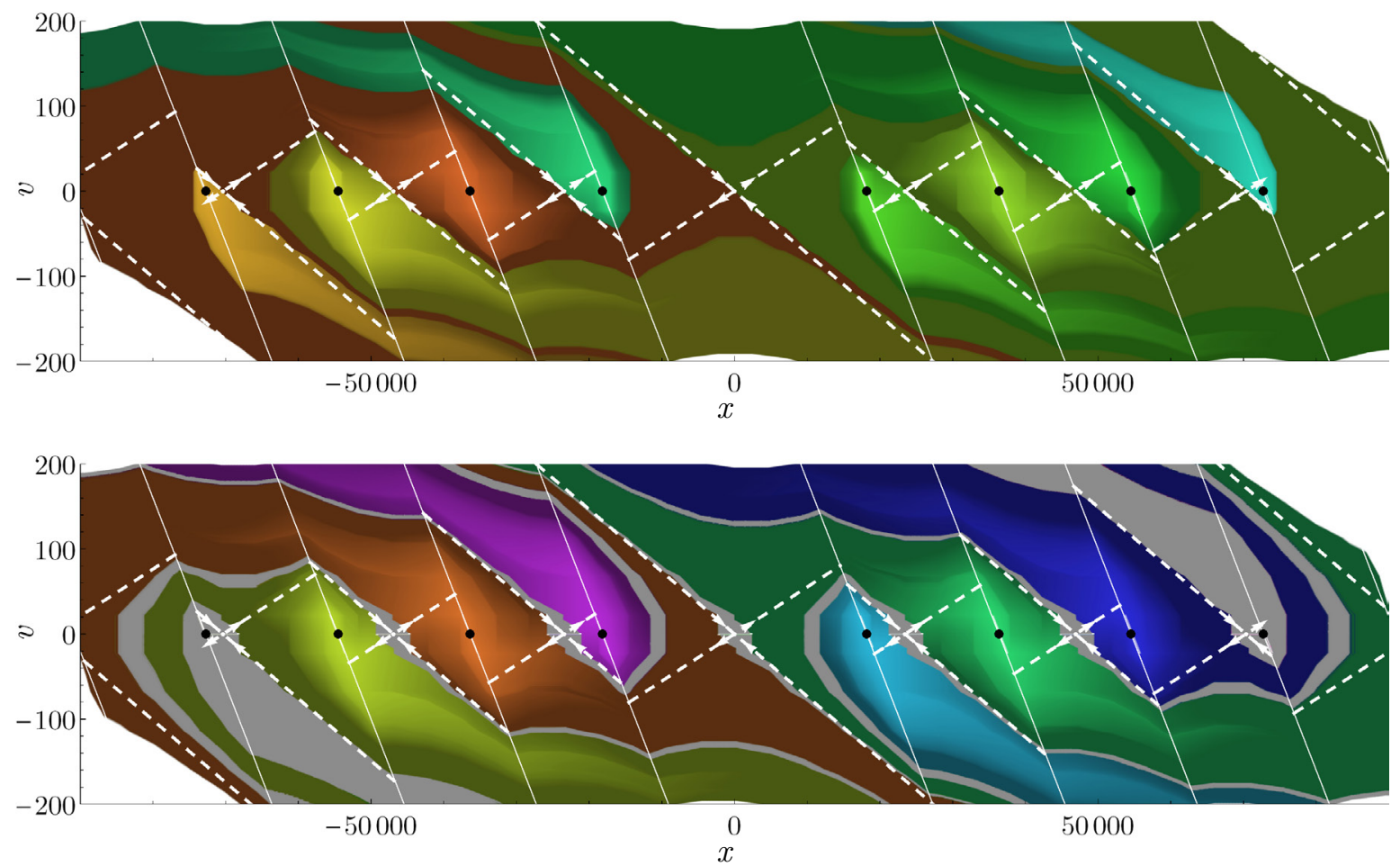

Fig. 4 C-SCM results for $\hat{\alpha}=6.53 \times 10^{-3}, \delta=0, \hat{P}=5.5 \times 10^{-5}, \hat{D}=2.5 \times 10^{-3}$, Top: $\hat{\mu}=0$, Bottom: $\hat{\mu}=0.095$. Chaotic attractors are indicated with black dots, their basins of attractions are colored regions and fixed points' manifolds are shown as white dashed lines. Gray regions indicate the basins of attractions of sticking zones, the bottom subfigure shows the case, when the outermost chaotic attractors are absorbed by sticking zones. 
Attractor position with respect to friction coefficient

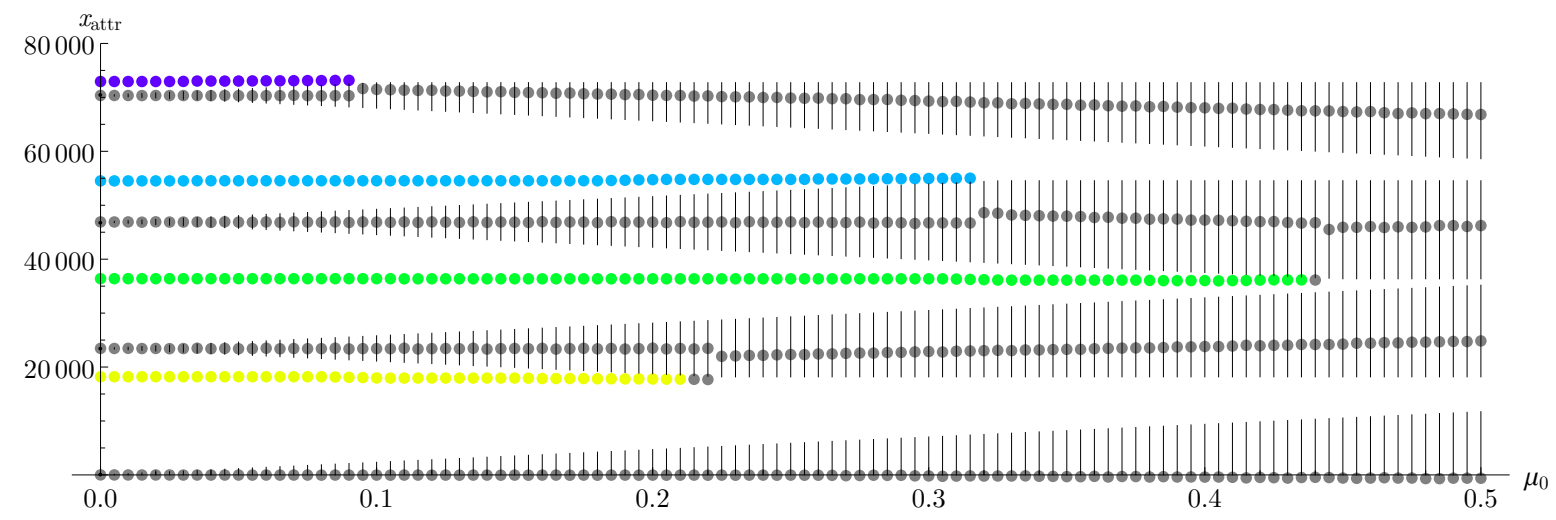

Fig. 5 C-SCM parameter scan results for $\hat{\alpha}=5.5 \times 10^{-5}, \delta=0, \hat{P}=5.5 \times 10^{-5}, \hat{D}=2.5 \times 10^{-3}, \mu_{0}=0 \ldots 0.5$. Colored points correspond to the location coordinate of chaotic attractors, while gray points indicate the centers of periodic cell groups corresponding to sticking zones. The theoretical boundaries of sticking zones are shown by vertical sections. As the friction parameter is increasing, chaotic attractors are absorbed by sticking zones, one by one.

originating from dry friction. Using the methodology introduced in this paper, one can explore the practically relevant ranges of system parameters by cell mapping and select parameters corresponding to a favorable state space configuration.

With a similar approach, other spontaneous switching phenomena, e.g., impact, could be taken into account, as well.

\section{References}

[1] Haller, G., Stépán, G. "Micro-chaos in digital control", Journal of Nonlinear Science, 6(5), pp. 415-448, 1996.

https://doi.org/10.1007/BF02440161

[2] Enikov, E., Stepan, G. "Microchaotic Motion of Digitally Controlled Machines", Journal of Vibration and Control, 4(4), pp. 427-443, 1998.

https://doi.org/10.1177/107754639800400405

[3] Csernák, G., Stépán, G. "Digital Control as Source of Chaotic Behavior", International Journal of Bifurcation and Chaos, 20(5), pp. 1365-1378, 2010.

https://doi.org/10.1142/S0218127410026538

[4] Gyebrószki, G., Csernák, G. "Methods for the Quick Analysis of Micro-chaos", In: Awrejcewicz, J. (ed.) Applied Non-Linear Dynamical Systems, Springer Proceedings in Mathematics and Statistics, Springer, Cham, Switzerland, pp. 383-395, 2014. https://doi.org/10.1007/978-3-319-08266-0_28

[5] Milton, J. G., Insperger, T., Cook, W., Harris, D. M., Stepan, G. "Microchaos in human postural balance: Sensory dead zones and sampled time-delayed feedback", Physical Review E, 98(2), pp. 022223-1 - 022223-7, 2018.

https://doi.org/10.1103/PhysRevE.98.022223

[6] Insperger, T., Milton, J. "Sensory uncertainty and stick balancing at the fingertip", Biological Cybernetics, 108(1), pp. 85-101, 2014. https://doi.org/10.1007/s00422-013-0582-2

\section{Acknowledgement}

This research was supported by the Hungarian National Science Foundation under Grant № NKFI 128422.

The research leading to these results has received funding from the European Research Council under the European Union's Seventh Framework Programme (FP7/2007-2013) ERC Advanced grant agreement № 340889.

[7] Stepan, G., Milton, J. G., Insperger, T. "Quantization improves stabilization of dynamical systems with delayed feedback", Chaos, 27(11), pp. 114306-1 - 114306-9, 2017.

https://doi.org/10.1063/1.5006777

[8] Csernák, G., Gyebrószki, G., Stépán, G. "Multi-Baker Map as a Model of Digital PD Control", International Journal of Bifurcation and Chaos, 26(2), pp. 1650023, 2016. https://doi.org/10.1142/S0218127416500231

[9] Csernák, G. "Quantization-induced control error in a digitally controlled system", Nonlinear Dynamics, 85(4), pp. 2749-2763, 2016. https://oi.org/10.1007/s11071-016-2859-1

[10] Widrow, B., Kollár, I. "Quantization Noise: Roundoff Error in Digital Computation, Signal Processing, Control, and Communications", Cambridge University Press, Cambridge, UK, 2008. https://doi.org/10.1017/CBO9780511754661

[11] Gyebrószki, G., Csernák, G. "Clustered Simple Cell Mapping: An extension to the Simple Cell Mapping method", Communications in Nonlinear Science and Numerical Simulation, 42, pp. 607-622, 2017. https://doi.org/10.1016/j.cnsns.2016.06.020

[12] Campbell, S. A., Crawford, S., Morris, K. "Friction and the Inverted Pendulum Stabilization Problem", Journal of Dynamic Systems, Measurement, and Control, 130(5), pp. 054502-1 - 054502-7, 2008. https://doi.org/10.1115/1.2957631 
[13] Deimling, K. "Multivalued Differential Equations", De Gruyter, Berlin, Boston, 1992.

https://doi.org/10.1515/9783110874228

[14] di Bernardo Laurea, M., Champneys, A. R., Budd, C. J., Kowalczyk, P. (eds.) "Piecewise-smooth Dynamical Systems: Theory and Applications", Applied Mathematical Sciences, Springer, London, UK, 2008. https://doi.org/10.1007/978-1-84628-708-4

[15] Popp, K., Stelter, P. "Stick-Slip Vibrations and Chaos", Philosophical Transactions of the Royal Society A: Mathematical, Physical and Engineering Sciences, 332(1624), pp. 89-105, 1990. https://doi.org/10.1098/rsta.1990.0102
[16] Insperger, T., Milton, J., Stepan, G. "Semidiscretization for TimeDelayed Neural Balance Control", SIAM Journal on Applied Dynamical Systems, 14(3), pp. 1258-1277, 2015. https://doi.org/10.1137/140975632

[17] Kruse, S., Hoffmann, N. P. "On the Robustness of Instabilities in Friction-Induced Vibration", Journal of Vibration and Acoustics, 135(6), pp. 061013-1 - 061013-8, 2013. https://doi.org/10.1115/1.4024939

[18] Hsu, C. S. "Cell-to-Cell Mapping: A Method of Global Analysis for Nonlinear Systems", Applied Mathematical Sciences, Springer, New York, USA, 1987.

https://doi.org/10.1007/978-1-4757-3892-6 